Int. J. Speleol. 10 (1978), pp. 185 - 193

\title{
Un Batrachuperus adulte dans une grotte d'Iran Espèce nouvelle?
}

\author{
par
}

\author{
M. CLERGUE-GAZEAU ${ }^{(1)}$ et J.P. FARCY(1)
}

Adult Batrachuperus in a cave in Iran. A new species?

SUMMARY

During a speleological exploration of a cave in Iran, a species of Urodele Hynobiidae was found. This Batrachian is either a new species of the genus Batrachuperus or an adult form of the species Batrachuperus persicus previously only described in its larval and juvenile forms. Certain observable differences suggest that it can be considered a new species.

Observations on its feeding habits indicate that the presence of this periodic trogloxene on the cave is not «accidental», but that it remains there for a long period during its life cycle.

\section{INTRODUCTION}

Limitée au Nord par la mer Caspienne et au Sud par la chaîne de l'Elbourz, la région septentrionale de l'Iran bénéficie d'un climat relativement tempéré, comparé à celui, désertique, des zones méridionales. Des forêts luxuriantes de hêtres y dominent, avec, plus à l'ouest, des aulnes et des érables. Du versant Nord du massif montagneux, descendent des rivières torrentielles venant se jeter dans la mer Caspienne, souvent après un parcours souterrain à travers la montagne; elles réapparaissent au pied de celle-ci en résurgences. Chaque ruisseau a creusé là, dans le calcaire, des galeries et des salles.

Au cours de l'exploration spéléologique de l'une de ces grottes en 1974, l'un de nous découvrit, sur un banc d'argile au bord de l'eau, un Urodèle adulte de forte taille - $23 \mathrm{~cm}$ - et le ramena en France, afin de l'etudier. La description et le comportement de ce Vertébré font l'objet du présent article.

La vaste cavité où se trouvait le Batracien, est située près de la route reliant les villes d'Ali Abad et de Gorgan, au Sud-Est de la mer, à l'altitude relative de 400 mètres par rapport à celle-ci. Elle comprend une longue galerie. La rivière, d'une largeur de 5 mètres et d'une profondeur d'environ 2 mètres débitait,

(1) Laboratoire de Zoologie, Université Paul Sabatier, 118 route de Narbonne, 31400 Toulouse. Manuscript reçu le $1^{\circ}$ mai 1978 
lors de la capture de l'animal, environ 10 1/s. Enfin, détail, important comme nous le verrons par la suite, la grotte abrite de nombreuses chauves-souris. La cavité s'élargit en un grand porche sur l'extérieur, le rüisseau sort par une résurgence et coule vers la Caspienne.

L'animal découvert au fond de la grotte à 200 mètres de l'entrée est une Salamandre de la famille primitive des Hynobiidae, se caractérisant par la fécondation externe d'oeufs contenus dans des sacs gélatineux plus ou moins allongés. On constate déjà dans l'oviducte la sécrétion d'une enveloppe commune, chaque oeuf entouré de sa propre enveloppe.

Les Hynobiidés adultes possèdent en outre un appareil hyobranchial avec deux paires d'épibranchiaux : le premier arc ayant l'épibranchial fusionné avec le cératobranchial, le deuxième arc les ayant libres. A l'intérieur de cette famille, notre Urodèle appartient au genre Batrachuperus, groupant des Salamandres dont l'habitat se situe dans les montagnes d'Asie centrale, et dont le centre géographique est le Setchouan et le Sikiang. Elles sont présentes jusqu'à 4000 mètres d'altitude et caractérisées par : des sillons costaux, des dents voméro-palatines disposées en deux séries transversales courtes et seulement quatre orteils.

Deux sortes de constatations nous intéressent: d'une part, sous sa forme adulte, on ne connaissait pas l'animal en Iran; d'autre part, au moment de sa capture, il séjournait dans une grotte.

I) aucun adulte du genre Batrachuperus n'était observé jusqu'alors dans cette région.

En effet, jusqu'à ces dernières années, nous notions la présence des genres Triturus et Neurergus, espèces du paléarctique occidental; par contre, aucune espèce du paléarctique oriental n'y était observée.

Lors d'un voyage en Iran, Steiner et Eiselt (1970) ont ramené des larves d'Hynobiidé. Comparées à celles des espèces connues, elles se présentaient comme une espèce nouvelle de Batrachuperus, nommée $B$. persicus.

Les auteurs les ont décrites en examinant leurs rapports avec les larves d'autres Batrachuperus notamment avec l'espèce la plus proche géographiquement, c'est à dire Batrachuperus mustersi (Afghanistan); cette dernière éloignée de plus de $2000 \mathrm{~km}$ des lieux de capture des larves de Steiner et Eiselt. En 1970, Schmidtler et Schmidtler entreprennent un voyage afin de découvrir l'adulte de l'espèce décrite à l'état larvaire; ils retournent aux mêmes lieux mais subissent un échec; cependant, ils ramènent vivantes de nouvelles larves de $B$. persicus et les élèvent jusqu'à l'état juvénile. Une troisième expédition en 1971 (Steiner 1973) se révéla aussi infructueuse que les précédentes: l'adulte restait introuvable et l'auteur concluait ainsi : «Adulte Tiere der Art führen in Sommer eine versteckte Lebensweise und sind weiterhin unbekannt».

Notre animal n'a pas été découvert au même endroit: les larves de Steiner et Eiselt proviennent d'un ruisseau extérieur, situé près de Assalam, dans le 


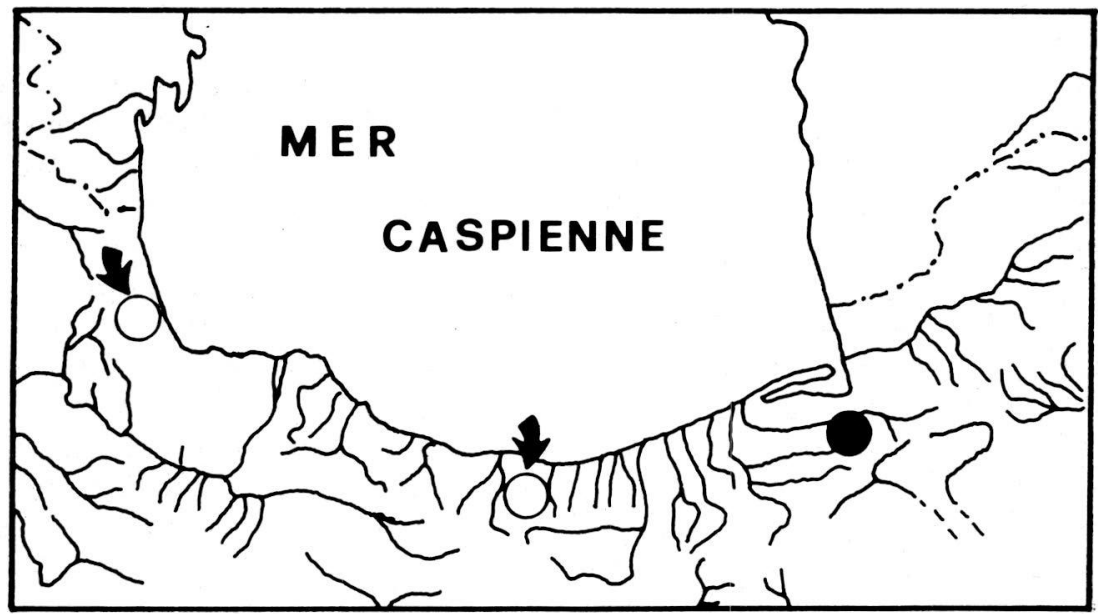

Fig. $n^{\circ} 1$ - Répartition géographique du genre Batrachuperus dans la région septentrionale de l'Iran.

Cercles blancs: Batrachuperus persicus (larves).

Cercle noir: Batrachuperus de Gorgan (adulte).

Nord-Ouest de l'Iran (fig. $\mathrm{n}^{\circ} 1$ ); quant à notre adulte, il vivait dans une grotte près de Gorgan, à l'Est de la Caspienne. A la dernière expédition, Steiner a reconnu au Sud un nouveau lieu de capture: cette station se situe à mi-chemin entre celle de Assalam et la grotte où se trouvait l'adulte. Posons nous maintenant la question: s'agit-il de la même espèce? Nous allons voir que, si l'adulte en notre possession ressemble par certains aspects morphologiques à l'espèce $B$. persicus il s'en éloigne par des rapports de mensurations et la forme de la tête. Nous ne pourrons nous prononcer valablement qu'après avoir comparé minutieusement l'adulte hypogé et les juvéniles issus des larves de Schmidtler et Schmidtler, dans un travail ultérieur plus approfondi. Pour l'instant, contentons nous de décrire la forme adulte de l'animal ramené d'Iran et de la comparer avec les espèces voisines.

\section{DESCRIPTION DE L'ADULTE MALE D'UNE ESPECE IRANIENNE DE BATRACHUPERUS.}

Tête déprimée, plus longue que large, à museau ovale. Grands yeux assez proéminents en position latéro-supérieure. Pli gulaire accentué, remontant légèrement latéralement sur les côtés du cou. Pas de lobes labiaux. Corps trapu, à tronc peu affaissé, marqué par une dépression vertébrale surtout prononcée à l'avant et comportant 10 sillons costaux. Queue plus longue que le reste du corps, de section sensiblement arrondie à la base et se comprimant rapidement vers l'arrière; l'extrémité en est pointue et relevée vers le haut (déformation?). 
Carène supérieure de la queue sur la totalité de sa longueur, prenant naissance au niveau des membres postérieurs, assez épaisse antérieurement, s'amincissant vers l'extrémité de la quelie. Pas de carène inférieure. Membres bien développés, les postérieurs robustes, se chevauchant légèrement lorqu'ils sont appliqués contre le tronc; orteils et doigts déprimés, s'amicissant vers l'extrémité arrondie; le premier orteil le plus petit, le troisième le plus long.

Absence de kératinisation sous les doigts et les orteils. Ouverture cloacale en fente longitudinale, longueur $6 \mathrm{~mm}$ ici, pour le mâle. Peau lisse, finement poreuse. Langue arrondie et très large. Nombre de dents voméro-platines: 11 dents courbées sur chaque série; petit intervalle séparant les deux séries dentaires voméro-palatines; ces deux séries sont en forme d'arcs et leur disposition ressemble à celle du genre voisin Onychodactylus (fig. $n^{\circ} 2$ ), à cette différence près que, chez Onychodactulus, les choanes se trouvent à l'avant, tandis que chez le Batrachuperus elles sont latérales par rappoii aux séries.

Coloration: autant que l'on puisse en juger sur un individu conservé dans l'alcool, la coloration est terne: marron sur le dos et jaune beige du côté ventral; nous sommes loin des teintes assez vives visibles sur la photographie du jeune métamorphosé, d'après l'article de Schmidtler.

Dimensions du sujet: longueur de la tête: $22 \mathrm{~mm}$,

largeur de la tête : $18 \mathrm{~mm}$,

longueur du tronc : $80 \mathrm{~mm}$,

longueur de la queue : $124 \mathrm{~mm}$.

Compte tenu du léger rétrécissement des tissus dû à l'alcool, on peut estimer la longuer totale à $230 \mathrm{~mm}$. Cette dimension est importante car les plus grands Hynobiidés ne dépassent pas la taille de $25 \mathrm{~cm}$.

Schmidtler et Schmidtler ont donné une description de l'espèce Batrachuperus persicus à l'état juvénile (environ $12 \mathrm{~cm}$ de longueur totale). Parmi les caractères communs nous paraissant les plus importants à mettre en évidence avec l'adulte en notre possession (et qui diffèrent nettement avec les autres espèces de Batrachuperus) citons : un pli gulaire accentué, des lobes labiaux non développés, des dents voméro-palatines en deux séries à peine séparées au milieu. Par contre, les différences les plus caractéristiques entre le juvénile de $12 \mathrm{~cm}$ et le subadulte de $15 \mathrm{~cm}$, d'une part, et l'adulte de $23 \mathrm{~cm}$, d'autre part, semblent porter sur : la taille, la position de yeux, la forme de la tête, la kératinisation, la coloration, la longueur de la queue et le nombre de sillons costaux.

1) La taille : $23 \mathrm{~cm}$ pour notre adulte, $15 \mathrm{~cm}$ pour celui de Schmidtler.

2) La position des yeux: les yeux de l'adulte de Gorgan sont en position latérosupérieure; par contre, ceux de l'individu de Schmidtler sont plus latéraux et plus antérieurs.

3) La forme de la tête: les rapports de diverses mensurations prises sur la tête : Longueur, largeur, distance du pli gulaire à l'oeil, à la naissance du mandibule 

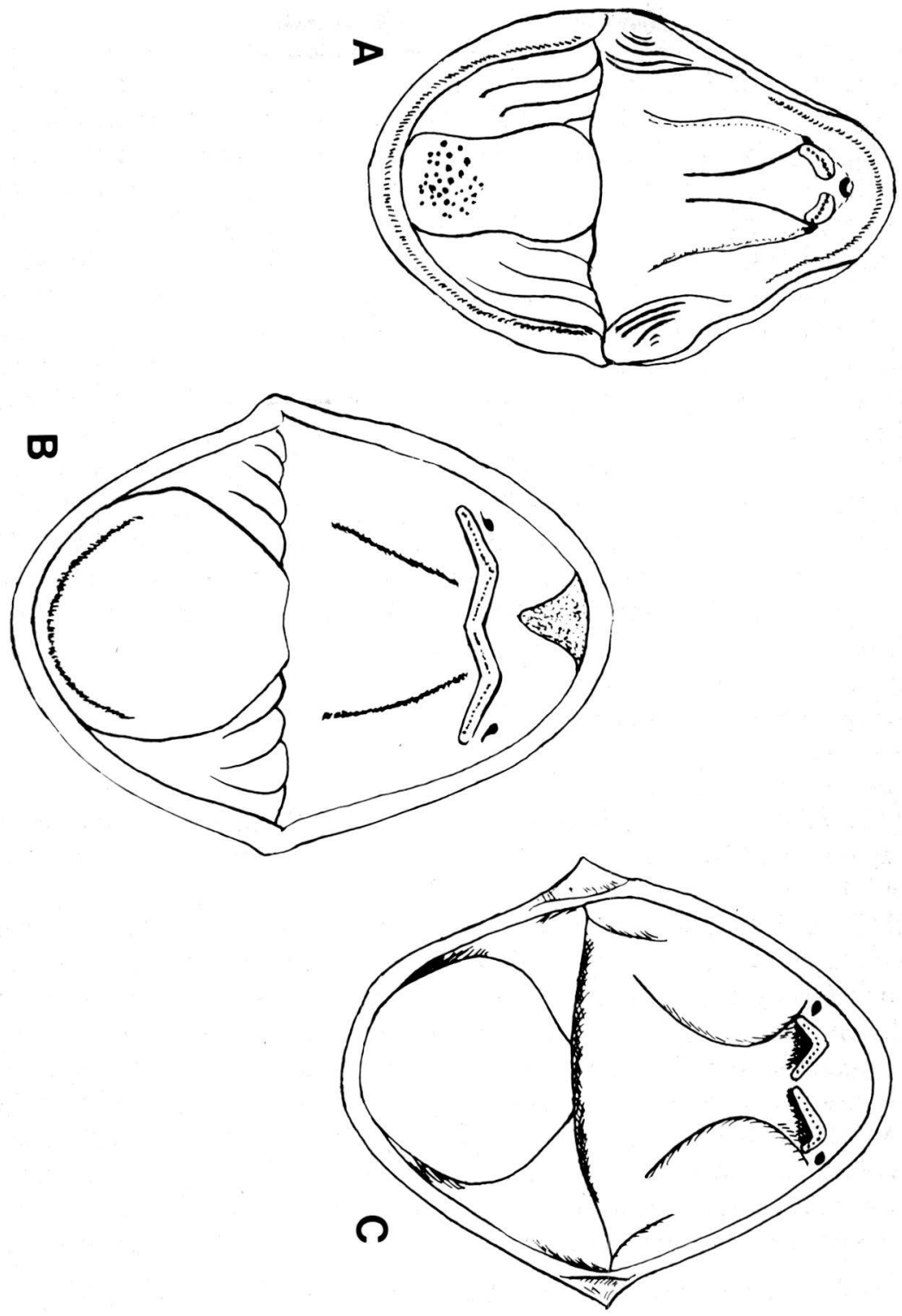

Fig. $\mathrm{n}^{\circ} 2$ - Schémas comparatifs de la position et de la forme des séries dentaires voméro-palatines et de la langue chez trois Urodèles hynobiidés.

A - Bouche ouverte de Batrachuperus yenyuanensis (d'après Liu).

B - Bouche ouverte de Onychodactylus japonicus (d'après Strejneger).

C - Bouche ouverte du Batrachuperus de Gorgan. 
etc...accusent une nette différence entre les exemplaires de Schmidtler et l'adulte hypogé.

4) La kératinisation: l'adulte de Schmidtler provenant des larves ramenées de Assalam montre une kératinisation nette sous les doigts et les orteils tandis que notre adulte n'en possède pas.

5) La longueur de la queue; la queue est bien plus longue que le reste du corps chez l'adulte: $12,3 \mathrm{~cm}$ au lieu de 10,2 (tête + tronc). Bien que l'écart dimensionnel soit important, à notre avis, ceci ne doit pas être retenu significativement; en effet, chez les Urodèles, la croissance caudale est très variable par rapport à celle du reste du corps (Clergue-Gazeau, 1972). Par exemple, chez l'espèce Eurucea longicauda longicauda, le pourcentage de la longueur du corps à la longueur totale du juvénile représente $44 \%$, tandis que chez l'adulte, il n'est que de 36,5\% ; autre exemple: chez Mertensiella caucasica ce pourcentage se monte à $54,6 \%$ pour la larve, $46,6 \%$ pour les juvéniles et $39,9 \%$ pour l'adulte. Les mesures de Steiner et Eiselt permettent de calculer quelques valeurs et de les comparer avec la nôtre;

larves de Assalam : 59\% pour une larve de $47 \mathrm{~cm}$

larves de Weiser: $58 \%$ pour une larve de $5,1 \mathrm{~cm}$

$51 \%$ pour une larve de $9,7 \mathrm{~cm}$

Juvénile de $12 \mathrm{~cm}$ : ?

Juvénile de $15 \mathrm{~cm}: 47 \%$

Adulte de $23 \mathrm{~cm}: 44 \%$

La diminution du pourcentage au fur et à mesure de la croissance chez Mertensiella caucasica (Salamandridae) et chez Eurycea l. longicauda (Plethodontidae), se retrouve chez notre Hynobiidé; par conséquent, la différence entre les juvéniles de Schmidtler et l'adulte étudié ne peut être retenue.

6) Le nombre de sillons costaux: cette différence nous apparaissait a priori comme bien plus significative: il nous semblait en effet que le nombre de sillons costaux ne variait pas lors de la croissance et était par conséquent, un élément caractéristique de l'espèce. Or, chez Batrachuperus persicus, l'évolution du nombre de sillons est le suivant: 14-15 chez les larves, 13-14 chez les juvéniles de $12 \mathrm{~cm}, 10-12$ à $15 \mathrm{~cm}$. L'espèce voit donc ses sillons costaux diminuer en nombre avec l'âge. Il se pourrait donc que ce nombre puisse se réduire définitivement à 10 chez d'adulte, comme pour notre exemplaire.

Quant à l'espèce Batrachuperus mustersi d'Afghanistan, elle diffère de l'individu en notre possession par: un pli gulaire presque indistinct, des lobes labiaux bien dévéloppés, une queue plus courte que le reste du corps et 8 à 9 dents au lieu de 11 sur chaque série de dents voméro-palatines.

II) Autre fait capital: lors de sa capture, l'animal se tenait dans une grotte, à une bonne distance de l'entrée - $200 \mathrm{~m}$ - dans l'obscurité totale. 
Première question à élucider: le Batrachuperus ne faisait il que passer dans la grotte ou y séjournait-il? pour un bref ou long séjour? propositions que nous allons examiner.

Tout d'abord son éloignement de l'entrée, sa position dans l'obscurité totale, suggèrent qu'il ne peut être question d'un passage «accidentel». L'état de conservation n'a pas permis un examen approfondi des yeux; néammoins, l'oeil protubérant, les pattes fortes et trapues indiquent qu'il ne s'agit pas d'un troglobie. Non transformé apparemment par la vie souterraine, l'animal nous réservait quelques surprises par son contenu stomacal et intestinal.

L'ouverture du tube digestif a donné lieu aux constatations suivantes:

a) le tube digestif était gonflé d'un bout à l'autre et ne présentait pas l'aspect habituel aplati et vide des hypogés;

b) ce qu'il renfermait variait peu, que ce soit dans l'estomac ou dans la partie postérieure de l'intestin; ce contenu se composait d'une grande quantité de débris de carapaces d'insectes: matériel disséqué, trituré, découpé, non reconnaissable, le tout mêlé à des poussières brunâtres: cet aspect fait penser à une nourriture à base de guano de Chauves-souris...

Or, nous avons déjà indiqué que la grotte abritait de nombreuses Chauvessouris: notre animal se nourrirait donc de guano; en effet, ce matériel composé de restes de carapaces aurait été au préalable dilacéré par les dents de ces Insectivores.

Trois observations viennent à l'appui de cette hypothèse:

1) le volume intestinal fait penser à un transit ininterrompu où seraient digérés au passage certains débris encore valables et des Invertébrés s'alimentant de guano (guanobies);

2) la quantité de débris de carapaces accumulés dénote une nourriture abondante, ce qui, en principe, n'existe pas dans une grotte;

3) la dentition du Batrachuperus ne lui permet pas de triturer ainsi les Insectes: les petites dents mandibulaires pointues, les dents voméro-palatines courbées, ont seulement la possibilité certainement, de maintenir la proie et de perforer, mains non de la découper aussi profondément.

D'autre part, il est peu vraisemblable que l'individu se nourrisse à l'extérieur car les débris trouvés dans l'estomac présentent le même aspect que ceux de l'intestin; avec des aliments pris au dehors, on pourrait identifier les animaux au moins dans l'estomac, ce qui n'est pas le cas.

En conséquence, de l'examen du mode d'alimentation de notre Batracien, nous pouvons conclure à la frequéntation de la grotte par l'animal durant une période assez longue, sans doute pendant les mois les plus chauds? ou les plus secs? Dans tous les cas, cela expliquerait pourquoi on n'a pas encore découvert d'adultes à l'état épigé; cette interprétation correspond exactement à l'idée de Steiner (1973) selon laquelle les adultes méneraient une vie cachée. Or, les expéditions signalées plus haut s'effectuaient toujours pendant l'été, 
période plus propre à la prospection, et, justement, à cette saison, les adultes devaient de réfugier dans les grottes en raison du climat, peut-être aussi pour y accomplir une période bien déterminée de leur cycle de vie à l'état hypogé. Steiner et Eiselt (1970) indiquent une temperature de l'air de $17^{\circ}$ par temps couvert et à $800 \mathrm{~m}$ d'altitude; compte tenu de l'adaptation du genre Batrachuperus aux températures très froides des montagnes du centre de la Chine, il apparaît que la température trop élevée du lieu de capture ne lui convient, pas et qu'il cherche un refuge à l'intérieur des grottes.

\section{CONCLUSIONS}

Jusqu'à ces dernières années, les espèces d'Urodèles connues en Iran appartenaient à la faune européenne du paléarctique occidental.

Les larves découvertes par Steiner et Eiselt, Schmidtler et Schmidtler, et Steiner montrent que les formes du paléarctique oriental s'y sont également installées. La présence au Nord-Est de l'Iran de notre animal confirme l'avancée vers l'Ouest de ce genre dont le centre de dispersion se trouve en Asie Centrale. Cette nouvelle station se situe sur une ligne que relie les lieux de capture des larves de Steiner et Eiselt aux habitats de la Chine. Elle nous renseigne sur le passage suivi par ce genre arrivant de l'Est, pour s'implanter dans cette région.

Nous avons pu décrire l'animal adulte. Seules des études ultérieures de son biotope et des alentours, ou une comparaison minutieuse avec les formes juvéniles et adulte des larves ramenées par Schmidtler et Schmidtler, permettront de conclure à l'existence, soit de Batrachuperus persicus en ces lieux, soit à celle d'une nouvelle espèce. Compte tenu des différences déjà enregistrées plus haut, il est vraisemblable de penser à la présence d'une espèce nouvelle. Nous nous garderons bien de nous prononcer sur la durée de la présence de cet adulte à l'intérieur de la grotte; par son aspect extérieur, on le classe dans les Trogloxénes; par son mode d'alimentation, il semblerait que le Batrachuperus ait l'habitude des séjours prolongés dans les cavernes puisqu'il se nourrit principalement de guano et de guanobies.

\section{RÉSUMÉ}

Découverte d'un Urodèle Hynobiidé au cours de l'exploration spéléologique d'une grotte, dans la région Nord-Est de l'Iran. Ce Batracien est, soit une nouvelle espèce du genre Batrachuperus soit la forme adulte de l'espèce Batrachuperus persicus décrite seulement à l'état larvaire et juvénile. Des différences nettes dans les rapports de mensurations font penser à une espèce nouvelle.

Par son mode de nourriture - absorption de guano - ce Trogloxène périodique nous indique que sa présence à l'intérieur de la grotte n'est pas «accidentelle», mais qu'il doit y séjourner pendant une période assez longue de son cycle de vie. 


\section{BIBLIOGRAPHIE}

CLERGUE-GAZEAU M., 1972 - Evolution du rapport longueur du corps/longueur totale pendant la croissance de l'Euprocte des Pyrénées (Batracien, Urodèle). Ann. Limnol., 8 (2) : 223-228. EISELT J. et STEINER H. M., 1970 - Erstfund eines hynobiiden Molches in Iran. Ann. naturhist. Mus. Wien, $74: 77-90$.

SCHMIDTLER J. J. et SCHMIDTLER J. F., 1971 - Eine Salamander - Novität aus Persien, $B a$ trachuperus persicus. Acquarien Mag, , 5 (11) : 443-445.

STEINER H. M., 1973 - Beiträge zur Kenntnis von Verbreitung, Okologie und Bionomie von Batrachuperus persicus. (Caudata, Hynobiidae). Salamandra Frankf., 9 (1) : 1-6.

THORN R., 1968 - Les Salamandres d'Europe, d'Asie et d'Afrique du Nord.

Ed. Lechevallier, Paris, 373 pp. 